\title{
Diarrheagenic Escherichia coli pathotypes and childhood diarrhea in the Bikaner region of Western Rajasthan
}

\author{
S. Barupal ${ }^{1}$, J. Lakhani ${ }^{2}$, D. Harwani ${ }^{3 *}$ \\ ${ }^{1,3}$ Department of Microbiology, Maharaja Ganga Singh University, Bikaner, Rajasthan, India \\ ${ }^{2}$ Department of Computer Science, Maharaja Ganga Singh University, Bikaner, Rajasthan, India \\ *Corresponding author: dharmesh@mgsubikaner.ac.in, Tel.: +91 8764131240
}

Available online at: www.isroset.org

Received: 03/Dec/2018, Accepted: 19/Dec/2018, Online: 31/Dec/2018

\begin{abstract}
Diarrhea caused by $E$. coli has been recognized as an important health problem among children in developing countries particularly in India and is a research priority of the diarrheal disease control program of the World Health Organization. Despite progress in preventing diarrhea and the spread of medical facilities in India, diarrhea remains the second leading cause of death in children, killing an estimated 321 children every day in 2015. It is the aim of the present research to gather information on current definitions, detection and prevalence, characterization of genetic determinants, virulence mechanisms, antibiotic resistance and epidemiology of the major DEC pathotypes with emphasis on the case studies conducted in the Bikaner region of Rajasthan in India. It has been found that the incidence of diarrheal diseases to be very high during the summer months followed by rainy or winter months in the Bikaner region of Rajasthan. Mode of water transportation, poor handling of water (at the household level), the presence of wastewater and unhygienic conditions predisposed children to diarrhea. In addition, indiscriminate stool disposal by mothers, lack of hand-washing before feeding their children or handwashing without soap has also been observed to be associated with the increased threat of diarrheal infection. The health associated risks of diarrheagenic E. coli in under-five children and the possible solutions to prevent the same are discussed.
\end{abstract}

Keywords-Diarrheagenic Escherichia Coli, Childhood diarrhea, Current definitions, Prevention and Control

\section{INTRODUCTION}

Diarrhea is estimated to be a leading cause of death globally among children under five years [1]. Despite progress in preventing diarrhea and the spread of medical facilities in India, diarrhea remains the second leading cause of death in children under five years of age, killing an estimated 321 children every day in 2015 [2]. Unfortunately, diarrhea kills more children than AIDS, malaria and measles combined [3]. The enteric pathogen diarrheagenic Escherichia coli (DEC) is the most common causes of diarrhea in children less than five years of age [4]. According to National Family Health Survey-4 [5] "region fact sheet", Bikaner, Rajasthan, the prevalence of diarrhea among the children under five years of age (in the last 2 weeks preceding the survey) has been found to be more than $10.0 \%$. Furthermore, a local survey to the city hospital in Bikaner region revealed that during the summer months the incidence and severity of diarrhea in children under five years of age increases drastically. A wealth of data concerning diarrheagenic Escherichia coli (E. coli; EC) infections has been accumulated over the years even though the complicated mechanism of its pathogenicity and virulence are not yet fully known. The section I of the present manuscript contains the introduction and present of the current scenario of childhood diarrhea in India and in Bikaner region of the Thar desert in Rajasthan, section II contains the information on diarrheagenic Escherichia Coli pathotypes and about the study area of present research and section III contains the need of research and possible solutions to control and prevent diarrheal infection in children under five years of age. Finally, section IV includes conclusion and future directions.

\section{DIARRHEAGENIC ESCHERICHIA COLI PATHOTYPES AND THE STUDY AREA}

Diarrheagenic E. coli (DEC) are classified according to their virulence factors and other phenotypic traits into enterotoxigenic E. coli (ETEC),

enteropathogenic E. coli (EPEC), Verotoxin or Shiga toxinproducing $E$. coli (VTEC/STEC) or enterohaemorrhagic E. coli (EHEC), enteroinvasive E. coli (EIEC), diffusely adherent E. coli (DAEC), and enteroaggregative E. coli (EAEC). Among the five known diarrheagenic E. coli ETEC, EHEC and EPEC are the most significant, with higher impact on countries of the developing world [6]. The contribution from the Bikaner region to the global knowledge regarding diarrheagenic enteropathogenic E. coli (EPEC), enterotoxigenic E. coli 
(ETEC), enterohemorrhagic E. coli (EHEC) and enteroaggregative $E$. coli (EAEC) including multi-drug resistant (MDR) pathotypes leading to childhood diarrhea seems very limited. A wide range of cell processes in children is affected by this pathogen via an array of diverse genetic elements enabling each diarrheagenic pathotype to colonize, multiply, and disseminate. The main problem is the fact that there is a large overlap (target genes and symptoms) between different $E$. coli pathotypes, which makes identification of the isolates difficult. Because of these difficulties, the focus has not been given on linking the specific E. coli (including multi-drug resistant (MDR) pathotypes) to the cases of childhood diarrhea. These gaps could be due to either insignificant clinical and scientific research or the paucity of well designed systematic epidemiological studies or the need for this was not recognized. Now, the importance of detection and identification of MDR diarrheagenic E. coli pathotypes has been recognized, mostly due to outbreaks of infections in children fewer than five years of age particularly in the summer season in the Bikaner region. The understanding of each pathogenic step may help in devising effective measures for prevention and control of diarrhea in children under five years of age. The region of Bikaner derives its name from its principal city "Bikaner" which was established by Rao Bikaji. Bikaner is one of the desert region situated in the North-West of Rajasthan. It extends from $27^{\circ} 11^{\prime}$ to $29^{\circ} 3^{\prime}$ North latitudes and $71^{\circ} 54$ to $74^{\circ} 12^{\prime}$ East longitudes. Bikaner region ranks 11 th in terms of population, 2nd in terms of area and 32nd in terms of population density. The major part of the region is comprised of desolate and dry regions which form part of the Great India Desert of Thar. Bikaner region has eight tehsils, in which Kolayat tehsil has the highest number of villages (232) whereas Khajuwala tehsil has the lowest number of villages (57) [7].

\section{THE NEED OF RESEARCH AND POSSIBLE SOLUTIONS}

The most $E$. coli strains live harmlessly in the colon and seldom cause disease in healthy individuals; a number of pathogenic strains can cause intestinal and extra-intestinal disease both in healthy and immuno-compromised individuals including children [8]. Diarrheal illness is a severe public health problem and a major cause of morbidity and mortality in infants and children under five years of age. Diarrhea caused by multidrug-resistant $E$. coli has been recognized as an important health problem among children in developing countries and is a research priority of the diarrheal disease control program of the World Health Organization. There are a widespread occurrence and prevalence of diarrhea childhood in Bikaner region of Rajasthan. This may be attributed to the poor living conditions, inadequate water supplies, poor environmental hygiene and sanitation, poor diagnosis and treatment and insufficient education etc [9], [10], [11]. According to the group of virulence determinants acquired, specific combinations were formed determining the currently known E. coli pathotypes, which are collectively known as diarrheagenic E. coli (DEC) [12]. The DEC pathotypes differ regarding their preferential host colonization sites, virulence mechanisms and the ensuing clinical symptoms and consequences, are classified as enteropathogenic E. coli (EPEC), enterohemorrhagic (Shiga toxin-producing) E. coli (EHEC/STEC), enteroaggregative $E$. coli (EAEC), enterotoxigenic $E$. coli (ETEC), and enteroinvasive $E$. coli (EIEC). Among DEC, enteropathogenic E. coli (EPEC), enterotoxigenic E. coli (ETEC), enterohemorrhagic E. coli (EHEC) and enteroaggregative $E$. coli (EAEC) involved in the diarrheal disease are the most important of the various etiological agents of diarrhea.

The MDR pathotypes have evolved by the acquisition, through horizontal gene transfer, of a particular set of resistant factors that have successfully persisted in the population [13]. Each of these pathotypes represents a group of clones that share specific virulence factors. Nevertheless, it should be pointed out that the plasticity of the $E$. coli genome has hindered the identification of certain $E$. coli isolates as a pathotype because some isolates combine the main virulence characteristics of different pathotypes and are thus considered potentially more virulent hybrid pathogenic strains [9]. DEC pathotypes require further epidemiological studies, which have been hampered by the difficulties in its identification and classification [14], [15]. DEC infections are an important health issue causing diarrhea among children under five years of age in Bikaner region of Rajasthan because of their association with high morbidity and mortality. The research methods are needed for the rapid detection of diarrheagenic enteropathogenic (EPEC), enterotoxigenic (ETEC), enterohemorrhagic (EHEC) and enteroaggregative (EAEC) as well as multidrug-resistant pathotypes and their antibiotic resistance genes responsible for diarrhea in children under five years of age of Bikaner region. Thus generated data will assist in finding the prevalence and taxonomic distribution of diarrheagenic $E$. coli pathotypes in the region. Identification of multidrug-resistant diarrheagenic E. coli pathotypes may help in providing more effective therapy to treat diarrhea in the affected regions and to design control measures. The genetic determinants and molecular mechanisms of diarrheal infections caused by diarrheagenic $E$. coli pathotypes may unlock innovative perspectives in the studies of diarrheal research, outcomes from which can be used for effective health care programmes for diarrhea prevention and management in the affected areas of not only Bikaner but also for other regions of Rajasthan.

\section{CONCLUSION AND FUTURE SCOPE}

The virulence mechanisms of diarrheagenic E. coli pathotypes have been accumulated over the years even 
though these complicated phenomena are yet to be understood. This versatile pathogen affects a huge range of cellular processes in eukaryotes using diverse genetic elements enabling each diarrheagenic pathotype to colonize, develop and disseminate. The understanding of each pathogenic step at the molecular level may help in devising effective measures to control its periodic outbreaks. However, the contribution from Bikaner region of Western Rajasthan in India to the global knowledge regarding these pathotypes seems very limited which could be due either to the insignificant systematic epidemiological, antimicrobial resistance research and absence of a surveillance system for diarrheagenic E. coli pathotypes. Therefore, research on to these aspects and various experiments are underway that may certainly help the national, regional, and zonal level policy makers, health institutions at each level to design and implement strategies to minimize diarrhea caused by diarrheagenic E. coli pathotypes in the children under five years of age.

\section{REFERENCES}

[1]. H.H., Kyu, C. Pinho, J.A. Wagner, J.C. Brown, A. Bertozzi-Villa et al., "Global and national burden of diseases and injuries among children and adolescents between 1990 and 2013: findings from the global burden of disease 2013 study", Global Burden of Disease Pediatrics Collaboration, JAMA Pediatrics, Vol. 170, pp. 267-287, 2016.

[2]. WHO, "http://www.who.int/en/news-room/factsheets/detail/diarrhoeal-disease", 2017.

[3]. WHO, "The Global Burden of Disease: 2004 Update", Geneva, 2008.

[4]. United Nations Children's Fund. "Diarrhoea: why children are still dying and what can be done", New York, NY: United Nations Children's Fund, p. 58, 2009.

[5]. NFHS-4, "http://rchiips.org/NFHS/pdf/NFHS4/RJ_FactSheet.pdf", 2015-16.

[6]. J.P. Nataro, J.B. Kaper, "Diarrheagenic Escherichia coli”, Clinical Microbiology Reviews, Vol. 11, pp.142-201, 1998.

[7]. Census India,“censusindia.gov.in/2011census/dchb/0803_PART_B_DCH B_BIKANER. pdf", 2011.

[8]. J.B. Kaper, J.P. Nataro, H.L.T. Mobley, "Pathogenic Escherichia coli”, Nature Review Microbiology, Vol. 2, issue 2, pp.123-140, 2004.

[9]. M.A., Croxen, R.J. Law, R. Scholz, K.M. Keeney, M. Wlodarska, B.B. Finlay, "Recent advances in understanding entericpathogenic Escherichia coli". Clinical Microbiology Review, Vol. 26, issue 4, pp. 822-880, 2013.

[10]. A.G. Abreu, C.M. Abe, K.O. Nunes et al., "The serine protease Picas a virulence factor of atypical enteropathogenic Escherichia coli", Gut Microbes, Vol. 7, issue 2, pp. 115-125, 2016.

[11]. T.A.T. Gomes, "Typical and atypical enteropathogenic Escherichia coli”, Emerging Infectious Disease, Vol. 8, pp. 508513, 2002.

[12]. J.P. Nataro, J.B. Kaper, "Diarrheagenic Escherichia coli", Clinical Microbiology Reviews, Vol. 11, pp. 142-201, 1998.

[13]. D. Vijay, P. Dhaka, J. Vergis, M. Negi, V. Mohan, M. Kumar et al., "Characterization and biofilm forming ability of diarrhoeagenic enteroaggregative Escherichia coli isolates recovered from human infants and young animals", Comparative
Immunology, Microbiology \& Infectious Diseases, Vol. 38, pp. 21-31, 2015.

[14]. A. von Mentzer, T.R .Connor, L.H. Wieler et al., "Identification of enterotoxigenic Escherichia coli (ETEC) clades with long-term global distribution", Nature Genetics, Vol. 46, pp.1321-1326, 2014.

[15]. A. von Mentzer, Thesis "Whole Genome Sequencing of Enterotoxigenic Escherichia coli (ETEC): Identification of ETEC Lineages and Novel Colonization Factors". Gothenburg, Sweden: Department of Microbiology and Immunology, Institute of Biomedicine, Sahlgrenska Academy at University of Gothenburg, 2016. 